\title{
ChemComm
}

Cite this: Chem. Commun., 2013, 49, 4504

Received 10th February 2013, Accepted 8th March 2013

DOI: $10.1039 / c 3 c c 41123 k$

\section{A new photoclick reaction strategy: photo-induced catalysis of the thiol-Michael addition via a caged primary amine $\dagger$}

\author{
Weixian $\mathrm{Xi}^{\mathrm{a}}{ }^{\mathrm{a}}$ Matthias Krieger, ${ }^{\mathrm{a}}$ Christopher J. Kloxin ${ }^{\mathrm{b}}$ and \\ Christopher N. Bowman*a
}

www.rsc.org/chemcomm

The utilization of 2-(2-nitrophenyl)propyloxycarbonyl (NPPOC) as a photolabile primary amine cage enables the thiol-Michael 'click' reaction to be photo-triggered. The photolabile amine exhibits efficient catalytic activity upon UV irradiation and is shown to initiate the photopolymerization of tetrathiol and diacrylate comonomers via Michael addition.

The thiol-Michael addition is one of the most widely implemented 'click' reactions in polymer science, being utilized in dendrimer synthesis and polymer network formation as well as bioconjugation and polymer functionalization. ${ }^{1-3}$ The thiolMichael reaction is readily catalyzed through either a base- and nucleophile-pathway; ${ }^{4,5}$ however, there are no light-mediated methods to control this reaction which would have a significant impact in several material fabrication strategies. Moreover, there are relatively few click reactions that are amendable to photoinitiation, such as the radical-mediated thiol-ene and photoreduced $\mathrm{Cu}$-catalyzed azide-alkyne cycloaddition reactions. ${ }^{2,6-8}$ Some commercially available photogenerated acids/bases have been used in many reactions such as thiol-epoxide reaction. ${ }^{9}$ This lack of utilization is likely due to the fact that their photolysis mechanism often includes the generation of radicals which would lead to acrylate (or other vinyl monomer) homopolymerization, rendering it inappropriate for Michael addition. Therefore, there is a need and challenge for developing novel light controlled reactions in both polymer chemistry and material science to expand and diversify the photo-mediated click reaction toolbox. In this communication, we introduce the concept of utilizing a photolabile catalyst for initiation of the thiol-Michael addition reaction to achieve spatiotemporal control of this highly versatile and implemented coupling reaction.

\footnotetext{
${ }^{a}$ Department of Chemical and Biological Engineering, University of Colorado, Boulder, Colorado 80309-0424, USA.E-mail: christopher.bowman@colorado.edu; Fax: +1-303-492-4341; Tel: +1-303-492-3247

${ }^{b}$ Department of Materials Science \& Engineering and Department of Chemical \& Biomolecular Engineering, University of Delaware, 150 Academy Street, Newark, Delaware 19716, USA

† Electronic supplementary information (ESI) available. See DOI: 10.1039/ c3cc41123k
}

The 2-nitrobenzyl functional group is perhaps the most common photolabile protecting group utilized in organic synthesis (e.g., $\mathrm{OH}, \mathrm{NH}_{2}, \mathrm{SH}, \mathrm{COOH}$, etc. $) .{ }^{10}$ In the last few decades, several 2-nitrobenzyl derivatives have been developed with better quantitative yields and photolytic efficiency. In particular, 2-nitroveratryloxycarbonyl (NVOC) and 2-(2-nitrophenyl)propyloxycarbonyl (NPPOC) have been used as primary amine protecting groups for spatioselective surface functionalization and photolithographic synthesis of oligo nucleopeptide/peptide microarray. ${ }^{11-17}$ Primary amines, such as hexylamine, are efficient catalysts for the thiol-Michael addition in macromolecular synthesis, but lack the spatiotemporal control afforded by photoinitiation.,18 In this study, we hypothesize that NPPOC will be an effective protecting group for hexylamine, which will be released upon UV irradiation to catalyze the thiol-Michael addition of a thiol and acrylate. We chose NPPOC as our photolabile functional group because of its high quantum yield during photolysis as compared with NVOC (Scheme 1). ${ }^{19}$

NPPOC-hexylamine was prepared by direct amidation and was subsequently photo-deprotected using 320-390 nm UV light (see ESI $\dagger$ ). The photolytic deprotection reaction of NPPOChexylamine was monitored by UV/Vis (Fig. 1). This photolytic reaction proceeded rapidly, finishing in 30 min based on

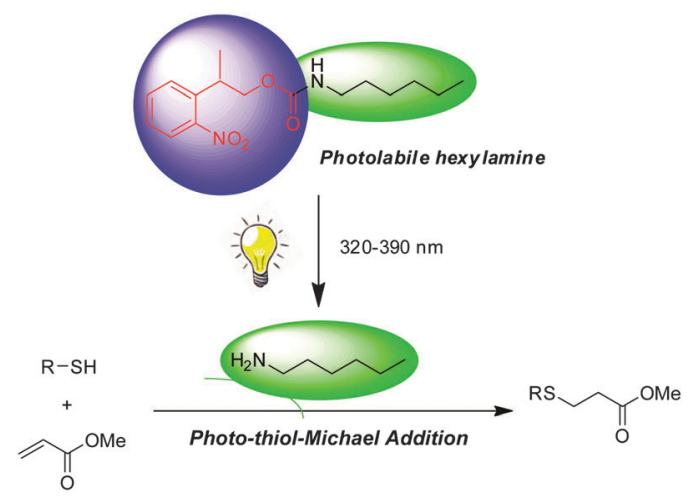

Scheme 1 Photo-triggered thiol-Michael addition reaction using NPPOC-hexylamine as a catalyst. 
(a)

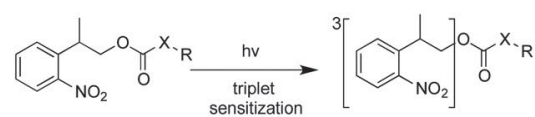

(b)

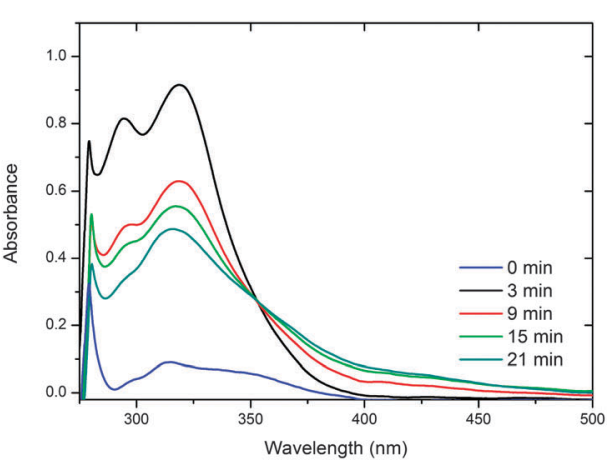

Fig. 1 (a) Principle photo-cleavage pathways and products of the NPPOCprotected substrates. (b) UV/Vis spectra of NPPOC-hexylamine $(0.05 \mathrm{mM})$ before and after photolysis using 320-390 nm irradiation in $\mathrm{MeOH}$.

UV/Vis spectroscopy. Previous researchers have elucidated two identical pathways for NPPOC photo-cleavage (Fig. 1a): the nitroso pathway, which usually occurs with 2-nitrobenzyl-type protecting groups, and $\beta$-elimination, which occurs in the presence of water or an amine base. ${ }^{15,16,20}$ The UV/Vis spectra reveal the initial formation of the nitroso product, having typical peak locations at 290 and $317 \mathrm{~nm}$ (Fig. 1b). These peaks then decrease with increasing irradiation time, which is attributed to the suppression of the nitroso pathway by the newly released hexylamine, in accordance with previous work. ${ }^{16}$ Thus, in the photolytic reaction of NPPOC-hexylamine, the $\beta$-elimination is favoured, which is further evidenced by ${ }^{13} \mathrm{C}-\mathrm{NMR}$ (see ESI $\dagger$ ).

To demonstrate the potential of NPPOC-hexylamine as a photocatalyst for the thiol-Michael addition reaction, we chose thiol glycolate and methyl acrylate as model substrates (Table 1). The model reaction gives over $90 \%$ yield in $1 \mathrm{~h}$, indicating this catalytic reaction proceeds rapidly and efficiently. Control experiments (entries 6 and 7 ) indicate that
Table 1 Scope of the NPPOC-hexylamine catalyzed thiol-acrylate addition ${ }^{a}$

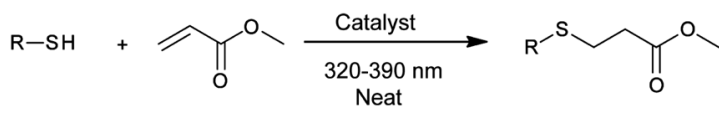

Catalyst:

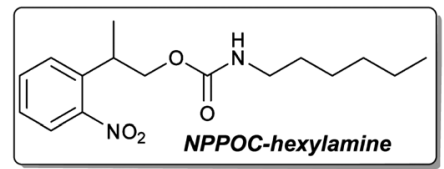

\begin{tabular}{|c|c|c|c|c|}
\hline Entry & $\mathrm{R}-\mathrm{SH}$ & $\begin{array}{l}\text { Catalyst } \\
\text { loading (mol\%) }\end{array}$ & $\begin{array}{l}\text { Irradiation } \\
\text { time (min) }\end{array}$ & $\begin{array}{l}\text { Yield }^{b} \\
(\%)\end{array}$ \\
\hline 1 & & 5 & 60 & 93 \\
\hline 2 & & 5 & 60 & 94 \\
\hline 3 & & 5 & 60 & 91 \\
\hline 4 & & 5 & 90 & 93 \\
\hline 5 & & 1 & 90 & 55 \\
\hline 6 & & 5 & $0^{c}$ & 0 \\
\hline 7 & & 0 & 90 & Trace \\
\hline
\end{tabular}

${ }^{a}$ Reaction conditions: thiol (1 mmol), methyl acrylate $(1 \mathrm{mmol})$ and NPPOChexylamine $(0.05 \mathrm{mmol})$ under $320-390 \mathrm{~nm}$ irradiation $\left(20 \mathrm{~mW} \mathrm{~cm}{ }^{-2}\right)$. ${ }^{b}{ }^{1} \mathrm{H}$-NMR yield. ${ }^{c}$ Irradiation time is $0 \mathrm{~min}$, reaction time is $120 \mathrm{~min}$.

both the photolabile catalyst and photo-irradiation are essential to trigger this reaction.

Real-time Fourier transform infrared (FTIR) spectroscopy reveals that the photo-induced catalysis of the thiol-Michael reaction proceeds in two stages (Fig. 2). The first stage is characterized by slow reaction kinetics, the duration of which exhibits a weak dependence on irradiation time. Notably, 2 and 4 minutes of irradiation show a similar, delayed upturn ( $\sim 6 \mathrm{~min}$ ) in the conversion trajectory towards the second stage of rapid reaction kinetics. As these are thin film specimens, these trends are not attributable to any thermally induced autoacceleration effect. One possible explanation is that the

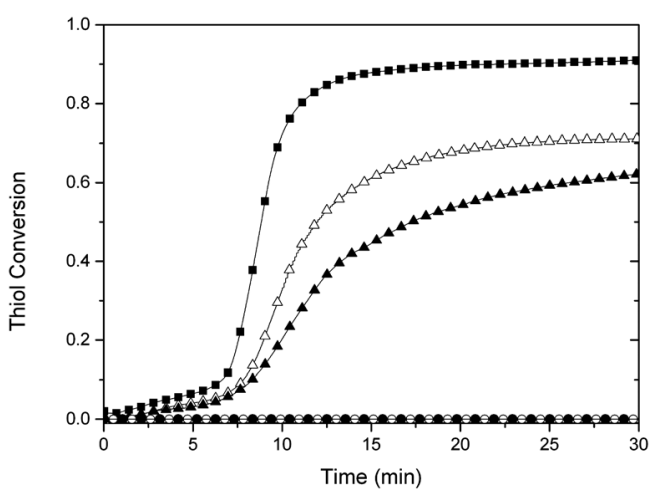

Fig. 2 Thiol conversion versus time, as measured by FTIR, for a stoichiometric mixture of butyl thiol glycolate and methyl acrylate using 5\% NPPOC-hexylamine under continuous (filled square), 4 min (open triangle), 2 min 320-390 nm irradiation (filled triangle), and no irradiation (open circle) as well as no NPPOChexylamine under continuous irradiation (filled circle). 
(a)

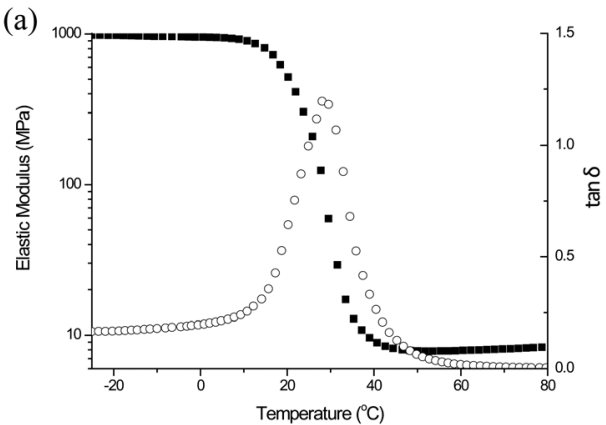

(b)

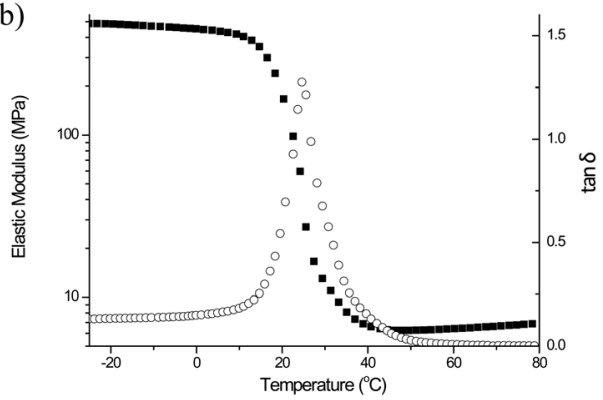

Fig. $3 \operatorname{Tan} \delta$ and elastic modulus plots vs. temperature for networks formed from a stoichiometric mixture of PETMA (a), PETMP (b) and bisphenol A ethoxy diacrylate.

conversion from the aci-nitro intermediate to the product is initially slow; however, upon the formation of the basic products (Fig. 1a), such as the newly released amine $\left(\mathrm{RNH}_{2}\right)$, thiol anion $\left(\mathrm{RS}^{-}\right)$, or carbon anion $\left(\mathrm{RCH}^{-} \mathrm{COOMe}\right)$, the deprotonatation of the aci-nitro intermediate pathway becomes favoured. As noted above, the $\beta$-elimination styrene product, which follows this reaction pathway, is observed in ${ }^{13} \mathrm{C}$-NMR (see ESI $\dagger$ ). Presuming that the kinetics of this nucleophileinitiated pathway is more rapid, combined with the increased basicity of the solution, the sudden increase in kinetics shown in Fig. 2 is explained. Additionally, no reaction occurs in the absence of irradiation, which in accordance with our ${ }^{1} \mathrm{H}-\mathrm{NMR}$ results.

Finally, we demonstrated the use of NPPOC-hexylamine as a photo-catalyst for the thiol-Michael photopolymerization reaction. We chose two tetrathiols (PETMA and PETMP) and a bisphenol A ethoxy diacrylate (BPAEDA) as substrates for the polymer network formation. To mitigate the side effects of $\mathrm{CO}_{2}$, which is released during photolysis, the NPPOC-hexylamine concentration was decreased from 5 to $3 \mathrm{~mol} \%$ and the irradiation time was extended to 1 hour. While similar, the resulting polymer networks exhibited a slight shift in glass transition temperatures as determined using dynamic mechanical analysis (DMA) via the peak in the $\tan \delta$ curve (i.e., 29 and $25^{\circ} \mathrm{C}$ for the PETMA/BPAEDA, Fig. 3a, and PETMP/BPAEDA, Fig. 3b, respectively). The larger

elastic moduli in the rubbery region for the PETMA- versus PETMP-based material suggests an increased crosslink density ( $0.95 \mathrm{M}$ versus $0.77 \mathrm{M}$ at $80^{\circ} \mathrm{C}$ ), which is attributed to the shorter distance between crosslinks in the PETMA system. In either case, the narrow $\tan \delta$ peaks indicate a homogeneous polymer network as observed in other thiol-ene photopolymerization reactions. ${ }^{2,3}$

In summary, we developed a photolabile, caged primary amine (NPPOC-hexylamine) as a photo-catalyst for the thiolMichael addition reaction and polymerization. This catalyst has efficient catalytic activity upon $320-390 \mathrm{~nm}$ irradiation. We demonstrated the utility of photolabile caged amines in photoinitiated thiol-Michael polymer network formation with the associated advantages of a homogeneous network with a sharp, well-defined glass transition.

This work was supported by the National Scientific Foundation grant CHE-1214109.

\section{Notes and references}

1 A. B. Lowe, Polym. Chem., 2010, 1, 17-36.

2 C. E. Hoyle, A. B. Lowe and C. N. Bowman, Chem. Soc. Rev., 2010, 39, 1355-1387.

3 C. E. Hoyle and C. N. Bowman, Angew. Chem., Int. Ed., 2010, 49, 1540-1573.

4 W. X. Xi, C. Wang, C. J. Kloxin and C. N. Bowman, ACS Macro Lett., 2012, 1, 811-814.

5 J. W. Chan, C. E. Hoyle, A. B. Lowe and M. Bowman, Macromolecules, 2010, 43, 6381-6388.

6 B. J. Adzima, Y. H. Tao, C. J. Kloxin, C. A. DeForest, K. S. Anseth and C. N. Bowman, Nat. Chem., 2011, 3, 256-259.

7 S. C. Ritter and B. Konig, Chem. Commun., 2006, 4694-4696.

8 J. W. Chan, H. Zhou, C. E. Hoyle and A. B. Lowe, Chem. Mater., 2009, 21, 1579-1585.

9 C. M. Seubert and M. E. Nichols, J. Coat. Technol. Res., 2010, 7, 615-622.

10 M. Zabadal and P. Klan, Chem. Listy, 2001, 95, 694-699.

11 K. R. Bhushan, C. DeLisi and R. A. Laursen, Tetrahedron Lett., 2003, 44, 8585-8588.

12 H. Giegrich, S. Eisele-Buhler, C. Hermann, E. Kvasyuk, R. Charubala and W. Pfleiderer, Nucleosides Nucleotides, 1998, 17, 1987-1996.

13 Z. C. Liu, D. S. Shin, K. T. Lee, B. H. Jun, Y. K. Kim and Y. S. Lee, Tetrahedron, 2005, 61, 7967-7973.

14 S. J. Min, K. D. Ahn and J. M. Kim, Bull. Korean Chem. Soc., 2005, 26, 1437-1439.

15 D. Woll, J. Smirnova, M. Galetskaya, T. Prykota, J. Buhler, K. P. Stengele, W. Pfleiderer and U. E. Steiner, Chem.-Eur. J., 2008, 14, 6490-6497.

16 H. Yi, S. Maisonneuve and J. Xie, Org. Biomol. Chem., 2009, 7, 3847-3854.

17 H. Zhao, E. S. Sterner, E. B. Coughlin and P. Theato, Macromolecules, 2012, 45, 1723-1736.

18 J. W. Chan, B. Yu, C. E. Hoyle and A. B. Lowe, Chem. Commun., 2008, 4959-4961.

19 A. Hasan, K. P. Stengele, H. Giegrich, P. Cornwell, K. R. Isham, R. A. Sachleben, W. Pfleiderer and R. S. Foote, Tetrahedron, 1997, 53, 4247-4264.

20 S. Walbert, W. Pfleiderer and U. E. Steiner, Helv. Chim. Acta, 2001, 84, 1601-1611. 